\title{
ДИФФЕРЕНЦИАЦИЯ НАНОПОРОШКОВ ПО РАЗМЕРАМ ЧАСТИЦ НА ПОПЕРЕЧНО КОЛЕБЛЮЩЕЙСЯ ПОВЕРХНОСТИ
}

\section{DIFFERENTIATION OF NANOPOWDERS ON THE SIZE OF PARTICLES ON A TRANSVERSELY VIBRATING SURFACE}

\author{
С.В.Каяашников", н.с. ^аборатории ФКМ ИФМ СО РАН, 2. Улан-Удэ, (ORCID: 0000-0003-3272-0072) / betch_kail@mail.ru \\ A.В.Номоев ${ }^{1}$, заведующий пабораторией ФКМ ИФМ СО РАН, г. Уяан-Удэ, д.ф.м.н., доцент, (ORCID: \\ 0000-0001-9400-3496) \\ S.V.Kalashnikov', Researcher of the FKM Laboratory of the Institute of Physical Materials Science, Siberian Branch of \\ the Russian Academy of Sciences, Ulan-Ude, (ORCID: 0000-0003-3272-0072) / betch_kail@mail.ru \\ A.V.Nomoev', Head of the FKM Laboratory of the Institute of Physical Materials Science, Siberian Branch of the \\ Russian Academy of Sciences, Ulan-Ude, Doct. of Sc. (Physics and Mathematics), Associate Professor, (ORCID: \\ 0000-0001-9400-3496)
}

DOI: $10.22184 / 1993-8578.2019 .12 .5 .250 .255$

Получено: 17.07.2019г.

Рассмотрены закономерности так называемого акустического метода разделения ультрадисперсных порошков - перераспределения частиц в узлы и пучности волны на поверхности поперечно колеблющейся пластины. Получены экспериментальные данные по эффективности разделения наночастиц диоксида кремния данным методом. Впервые выявлена зависимость характерного размера разделения частиц от амплитуды колебаний, меньших, чем глубина проникновения акустических вязких волн.

The regularities of the acoustic method of separation of ultrafine powders - the redistribution of particles into nodes and the antinodes of a wave on the surface of a transversely oscillating plate - are considered. Experimental data on the efficiency of the separation of silica nanoparticles by this method were obtained. For the first time, the dependence of the characteristic size of the particle separation on the amplitude of oscillations smaller than the thickness of the Stokes boundary layer was revealed.

\section{ВВЕДЕНИЕ}

Применение порошковых материалов в науке и технике широко распространено, особенно в материаловедении, металлургии, микроэлектронике, аналитической химии, биохимии и др. Все более широко наноразмерные порошки используются в различных технологиях, разработано множество методов получения данных материалов, которые, в большинстве своем, дают продукт с широким гранулометрическим составом. Частицы различного размера имеют, как правило, разные свойства, которые не могут быть адекватно изучены и технологически применены, пока частицы не разделены на отдельные фракции с узким распределением по размеру. Важно, например, определение наиболее оптимального размера частиц нанопорошка для модифицирования металлов [1], полимеров [2], эмалей и резин [3].

Ввиду очень малого размера и массы частиц нанопорошков, известные методы разделения по размерам частиц имеют ограниченные возможности. Если говорить о существующих методах разделения наночастиц, то следует отметить следующие: седиментация [4], фильтрование, абсорбционные методы, грохотание, электромагнитное разделение, броуновская диффузия, вибрационная 
сегрегация, гель-электрофорез и диэлектрофорез, аэродинамическое разделение, центрифугирование [5]. Закономерности и особенности перечисленных методов известны. Аэродинамический метод, как правило, пока используется только для разделения изотопов урана и, по сути, является видом центрифугирования.

Что касается рассматриваемого акустического разделения, то оно является новым и перспективным методом, однако использование этого способа для наноразмерных систем еще недостаточно изучено. Этот метод основан на фигурах Хладни, построенных частицами порошка на поперечно колеблющейся пластине, где частицы пространственно разделяются в пучности или узлы стоячих волн в зависимости от размера. Первые упоминания об использовании микрофигур Хладни для разделения наноразмерных материалов содержатся в [6], однако при очень высоких частотах и, соответственно, с малыми размерами колеблющейся пластины $\left(560 \times 100 \times 7 \mathrm{M} \mathrm{KM}^{3}\right)$, что ограничивает применение в прикладных задачах. Обнадеживающие результаты по разделению нанопорошка диоксида кремния акустическим методом при низких частотах получены нами и опубликованы в [7].

Физические причины распределений частиц по размерам связаны с потоками воздуха или другой среды, находящейся над пластиной. В последнее время появился интерес к данным потокам в рамках создания мини-вентиляторов в виде вибрирующих пластин для микроэлектронных устройств [8] в качестве средства манипулирования частицами в рамках различных приложений [9]. В работе [6] авторы пришли к выводу, что за движение частиц к пучности отвечает приповерхностное вихревое течение Шлихтинга [10], имеющее высоту своего центра от пластины, равную глубине проникновения вязких акустических волн. На частицы, размер которых меньше, действует только поток вблизи поверхности колеблющейся пластины и в направлении узла; для частиц с размером больше - потоки вихря Шлихтинга в целом и поток нижней части вихря Рэлея, которые приводят частицу к пучности. Следовательно, для разделения частиц с размером 100 нм $(\delta=100$ нм) в воздухе необходима очень высокая частота механических колебаний - 1 ГГц, что ограничивает практическую ценность метода во многих приложениях. Как уже упоминалось выше, нами в работе [7] обнаружено разделение частиц с размером менее 100 нм акустическим методом при низкой частоте 2 кГц, в отличие

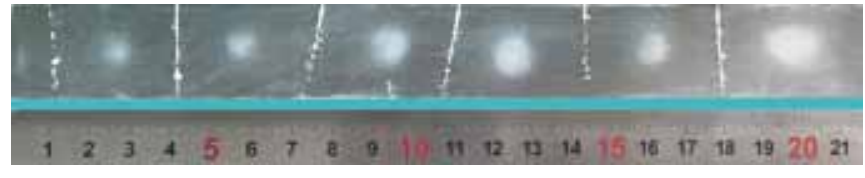

Puс.1. Поперечно колеблющаяся пластина с нанопорошком диоксида кремния на поверхности, образовавшим фигуры Хладни Fig.1. A transversely oscillating plate with silicon dioxide nanopowder on its surface, forming Chladney figures

от высокочастотных колебаний [6], при которых концентрация частиц меньшего размера инвертируется - происходит в пучности колебаний. Данное явление в настоящее время не полностью изучено и требует исследований с целью выявления закономерностей разделения и объяснения его причин, чему и посвящена данная работа.

\section{МЕТОДЫ ИССЛЕДОВАНИЯ}

Для исследования разделения нанопорошка по размерам частиц использовалась экспериментальная установка, представляющая собой консольно закрепленную горизонтальную стальную пластину (кантилевер) на подвесе электромагнитной системы, возбуждающей поперечные механические колебания, и питающуюся от генератора звуковой частоты. Амплитуда колебаний пластины измерялась оптическим методом. В экспериментах, проводившихся в воздушной среде, на поверхность пластины равномерным слоем распылялся нанопорошок диоксида кремния "Таркосил" с исходным средним размером частиц 28 нм. В случае применения в качестве среды жидкости, пластина помещалась в прямоугольный стеклянный сосуд объемом 10 л, заполненный диспергированной ультразвуком суспензией нанопорошка в воде с малой концентрацией 0,05 масс. \%. Ультразвуковая диспергация и малая концентрация порошка позволяла получить в воде стабильную суспензию с практически изолированными друг от друга частицами, что показал анализ суспензии методом рассеяния света. При колебании пластины на ее резонансной частоте порошок перераспределяется в узловые линии, и в виде круглых "облаков" - на пучности колебаний (рис.1).

Для анализа размера частиц порошок из различных областей отбирался путем засасывания тонким потоком воздуха, создаваемого дозатором пипеточным (модели дПОП-1-100-1000) со специальной насадкой. Гранулометрический состав порошков измерялся методом рассеяния света на приборе SALD-7500. 


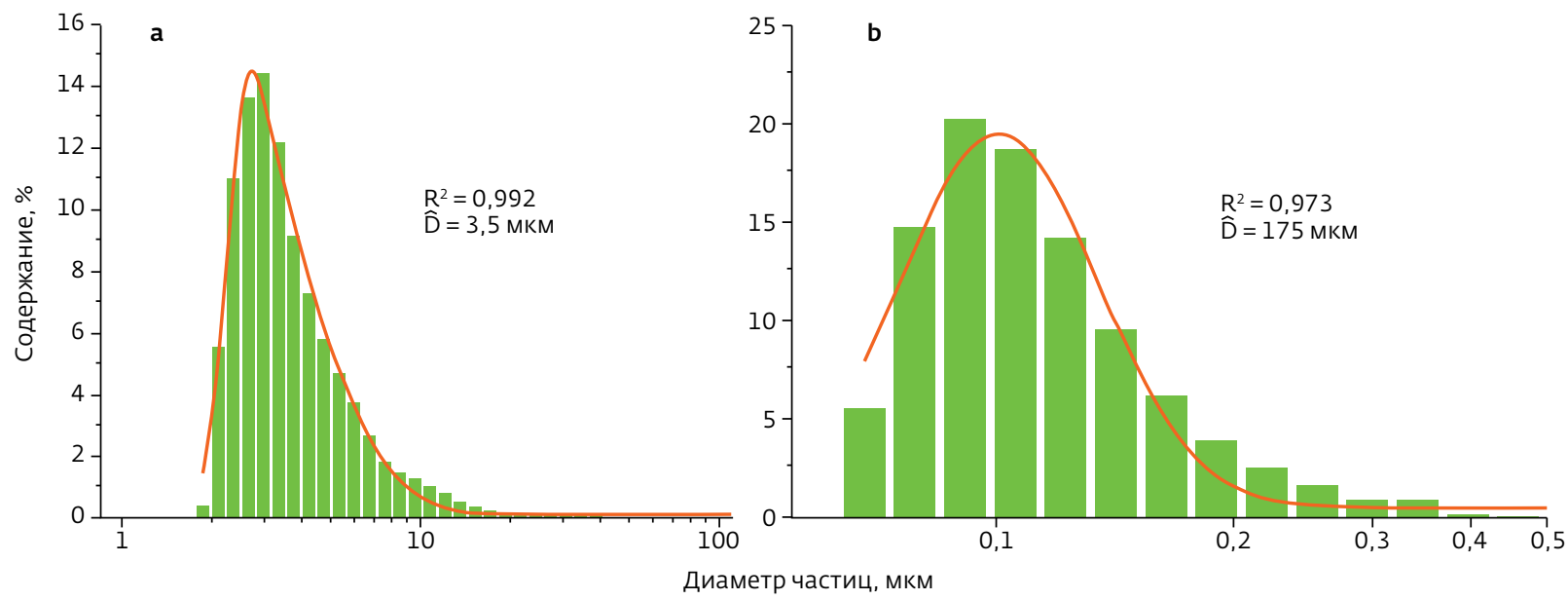

Puс.2. Распределение по размерам нанопорошка, взятого: $а$ - из узловой пинии; б - из пучности колебаний пластины Fig.2. Nanopowder size distribution, taken: $a$-from the nodal line; $b$-from the antinodes of the plate oscillations

\section{ЭКСПЕРИМЕНТАЛЬНЫЕ РЕЗУЛЬТАТЫ}

В экспериментах в воздушной среде при различных резонансных частотах $(0,47$ и 1,035 кГц) и при амплитуде колебаний $1 \cdot 10^{-4}$ м устойчиво наблюдается меньший размер частиц в пробах, взятых из пучностей (рис.2), притом средний размер проб порошков из пучности и узла колебаний существенно отличается. Распределения хорошо аппроксимируются кривой Гаусса, то есть вид распределения при разделении не изменяется.

В результате серии экспериментов была выявлена закономерность между амплитудой колебаний пластины и средними размерами частиц порошка, отобранного из узловых линий и из пучности колебаний (рис.3). Средние размеры наночастиц, отобранных из пучности, значительно

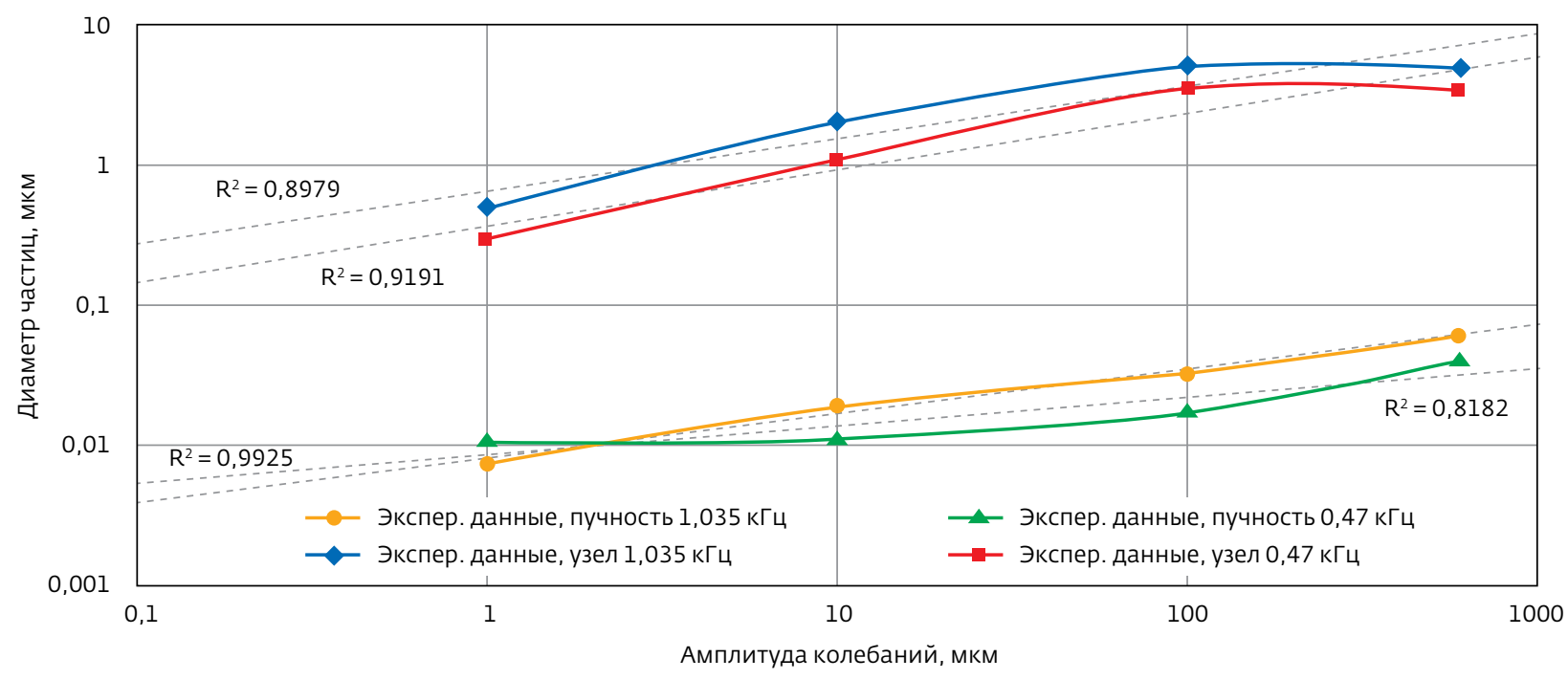

Pис.3. Зависимость среднего диаметра частиц диоксида кремния, отобранных из узловых пиний и из пучностей колеблющегося в воздухе кантилевера с частотой 0,47 и 1,035 кГи

Fig.3. Dependence of the average diameter of silicon dioxide particles selected from the nodal lines and from the antinodes of a cantilever oscillating in air with a frequency of 0.47 and $1.035 \mathrm{kHz}$ 
меньше размера частиц из узловой линии, при этом с увеличением амплитуды растет и средний диаметр частиц обеих фракций. Средний размер частиц, отобранных из узловых линий, при значениях амплитуды от 100 мкм и выше не изменяется, что может быть объяснено максимальным значением размера конгломератов порошка диоксида кремния в воздухе для данного эксперимента (3,5-5 мкм).

В эксперименте, проведенном на пластине, колеблющейся в воде, в которой были взвешены диспергированные ультразвуком наночастицы диоксида кремния, не было выявлено сепарации по размерам частиц. Все экспериментальные кривые средних размеров частиц, отобранных в узлах и пучностях колебаний, накладываются друг на друга (рис.4), при этом в среднем диаметр частиц равен исходному для примененного нанопорошка, то есть около 28 нм.

В воздушной среде наибольшая возможная амплитуда колебаний составляла 600 мкм, при большем значении фигуры Хладни размывались из-за большой кинетической энергии, передаваемой частицам от пластины. В воде фигуры были устойчивы и при амплитуде, равной 1 мм, что объясняется ее большей вязкостью. При амплитуде колебаний пластины, меньшей 1 мкм, фигуры не образовывались в обеих средах ввиду малой кинетической энергии пластины и, как следствие - малой скорости потоков среды.

\section{ОБСУЖДЕНИЕ}

Полученные экспериментальные результаты разделения наночастиц по размерам в воздушной среде могут быть объяснены следующим образом.

Во-первых, скопление мелких частиц на пучности колебаний, а не узле, говорит о том, что направление обращения вихрей среды над колеблющейся поверхностью обратно направлению, установленному исследователями в работе [6]. Это также подтверждается моделированием потоков, проведенных в публикации [11], где обнаружено обратное направление потоков Рэлея при частотах 60-1000 Гц.

Во-вторых, вид фигур Хладни зависит от отношения средней силы Стокса, действующей на частицы со стороны потоков среды над колеблющейся пластиной, к силе тяжести, действующей на частицу [12]. При выполнении для отдельных частиц условия В $>1$, эти частицы увлекаются потоком среды над колеблющейся пластиной (нижней частью вихря Шлихтинга)

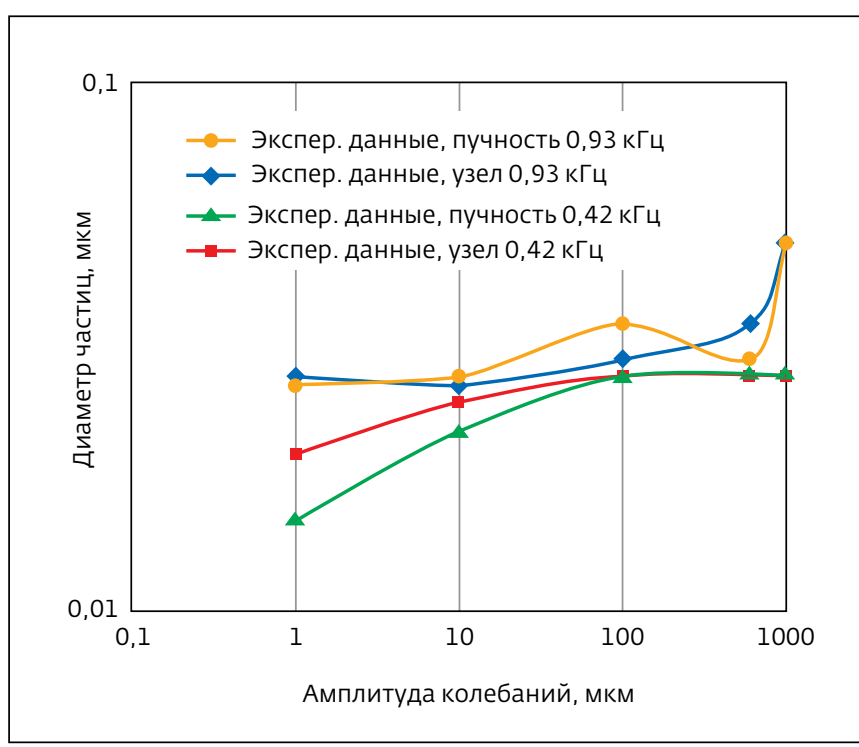

Рис.4. Зависимость среднего диаметра частиц диоксида кремния, отобранных из узловых пиний и из пучностей коиеблющегося в воде кантилевера с частотой 0,42 и 0,93 кГи Fig.4. Dependence of the average diameter of silica particles selected from the nodal lines and from the antinodes of a cantilever oscillating in water with a frequency of 0.42 and $0.93 \mathrm{kHz}$

и сосредотачиваются на пучности. Если $\mathrm{B}<1$, движением частиц управляет сила тяжести: в результате колебаний пластины частицы подпрыгивают, последовательные прыжки имеют тенденцию увеличивать кинетическую энергию частиц, однако в узловых линиях у пластины нулевая скорость и столкновения с ней, наоборот, уменьшают кинетическую энергию. В результате, большинство частиц скапливается в узловых линиях, формируя стандартные фигуры Хладни. Так как сила Стокса описывается выражением $\mathrm{F}_{\text {Stocs }}=6 \pi \mathrm{r} \mu \mathrm{v}$ и зависит от радиуса частицы $r$, вязкости среды $\mu$ и от ее скорости v, а сила тяжести - от плотности р и радиуса частицы r, то имеет смысл определить функцию, выражающую зависимость критического радиуса частицы, при котором она может быть сосредоточена в пучности колебаний (то есть при В=1) от вязкости среды, частоты и амплитуды колебаний. Так как скорость среды можно приблизительно представить в виде $\mathrm{v} \sim \alpha \omega$, где A - амплитуда, $\omega$ - циклическая частота колебаний и $\alpha$ - некоторый коэффициент пропорциональности, и, переходя от радиуса частицы к ее диаметру d, получим выражение:

$$
\mathrm{B} \sim 18 \mu \alpha \mathrm{A} \omega / \mathrm{d}^{2} \rho \mathrm{g} .
$$




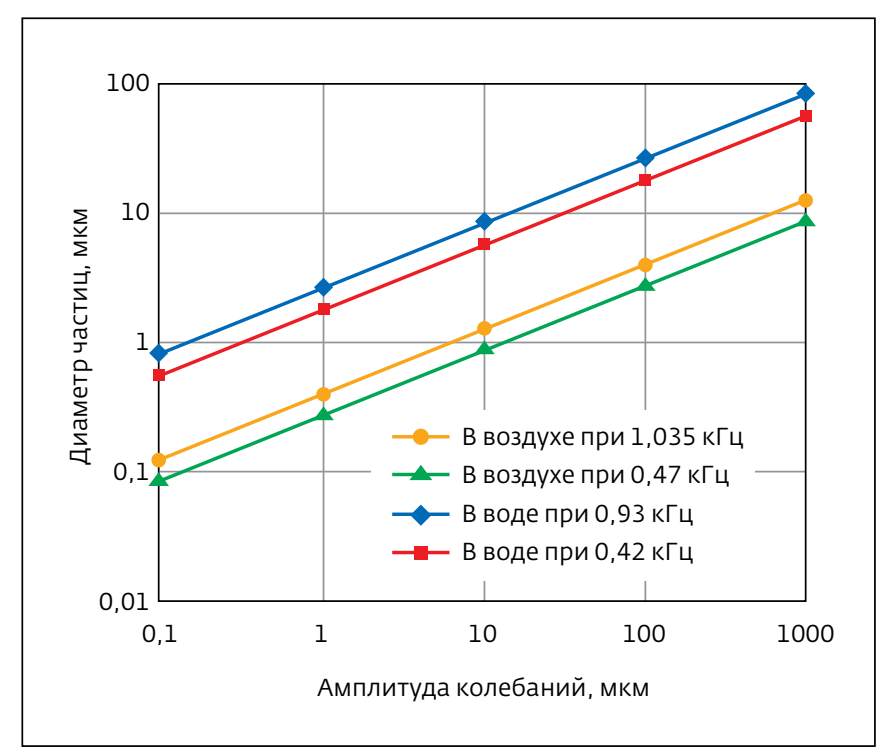

Pис.5. Зависимость диаметра частиц диоксида кремния от амплитуды колебаний при различных частотах в воздухе и воде (теоретические данные критического диаметра частиц (меньше которого частицы сосредотачиваются в пучности, большего - в узле). Обе шкалы логарифмические Fig.5. Dependence of the diameter of silicon dioxide particles on the amplitude of oscillations at different frequencies in air and water (theoretical data of the critical particle diameter (smaller than which particles concentrate in an antinode, larger - in a node). Both scales are logarithmic

При В=1:

$$
\begin{aligned}
& 18 \mu \alpha \mathrm{A} \omega \mathrm{d}-2 \rho g \\
& \mathrm{~d} \sim 3 \sqrt{\frac{2 \mu \alpha \mathrm{A} \omega}{\rho g}} .
\end{aligned}
$$

Коэффициент между скоростью среды и произведением определен исходя из данных о средней скорости среды в численной модели [11] при определенных значениях амплитуды и частоты колебаний пластины. Графически функция (2) для нанопорошка диоксида кремния приведена на рис. 5 в виде зависимости диаметра частиц (пограничное значение диаметра) при меньшем размере частицы будут сосредоточены на пучности колебаний, при большем - в узлах) от амплитуды колебаний при различной частоте и в двух средах - воздухе и воде соответственно. Видно, что данные теоретические прямые для воздушной среды находятся между экспериментальными кривыми среднего размера частиц, отобранных из пучности и из узла колебаний (см. рис.3). Причем в экспериментальных данных, как и в теоретических, при меньшей частоте достигается меньший размер разделяемых частиц. Прямые аппроксимации степенной функцией данных экспериментов на рис.3 по наклону достаточно близки к теоретическим прямым, приведенным на рис.5 (следует помнить, что графики приведены в логарифмических координатах, и термины "прямые" и "кривые" применимы лишь с визуальной точки зрения).

Разделение возможно лишь в приграничном слое толщиной $\delta$, этим объясняется уменьшение среднего диаметра частиц, отобранных из узловых линий и его увеличение у частиц, отобранных из пучностей колебаний для нанопорошка, разделенного в воздушной среде (рис.3) при амплитуде, большей 70-100 мкм, то есть амплитуде, равной или большей толщины проникновения вязких волн при данной частоте (для воздуха при 1 кГц $\delta=70$ мкм; при 0,5 кГц $-\delta=100$ мкм). Другими словами, когда амплитуда колебаний пластины становится сопоставима или больше толщины проникновения вязких волн $\delta$, разделение теряет свою эффективность - средний размер обеих фракций частиц приближается по значению друг к другу. Это вызвано тем, что при высокой амплитуде частицы, находящиеся на пучности, поднимаются пластиной в более высокие слои обращения вихревого течения Шлихтинга, где оно имеет направление потока в сторону узла колебаний, увлекаясь которым, малые частицы движутся в узел к частицам большего размера, то есть разделение по размерам прекращается.

Отсутствие разделения в воде можно объяснить тем, что за счет диспергирования количество конгломератов частиц и их размер стали невелики, большая часть частиц за счет низкой концентрации в воде находятся в изолированном состоянии и имеют диаметр, много меньший пограничного размера разделения для воды (из рис.5 - в -100-1600 раз). Что касается работ [7, 13], где разделение производилось в воде на поверхности пьезоэлемента с частотой 2 кГц, то обнаруженное там разделение объясняется тем, что порошок не был диспергирован, обладал большой концентрацией в воде и потому большим количеством и размером конгломератов, которые и определили состав фракции в узлах колебаний.

\section{выводы}

Таким образом, экспериментальные данные разделения нанопорошка на поперечно колеблющейся поверхности показывают, что разделение возможно при сравнительно низких частотах 
(0,5-1 кГц), то есть когда размер разделяемых частиц много меньше толщины слоя $\delta$ приповерхностного течения, при этом частицы меньших размеров скапливаются в пучности колебаний, а более крупные - в узловых линиях. Выявлено наличие зависимости размера частиц во фракциях из пучности и узлов колебаний не только от частоты колебаний, но и от амплитуды. Последняя особенно важна при низкой частоте, так как размер разделенных частиц при этом может регулироваться изменением амплитуды, что важно с точки зрения применения акустического метода разделения в прикладных целях.

Уменьшение размера разделяемых частиц возможно путем уменьшения амплитуды колебаний, однако существует нижний предел значения амплитуды, меньше которого фигуры Хладни не образуются ввиду малости колебательной энергии. Тем не менее в проведенных экспериментах удалось получить частицы малой фракции со средним размером 75 нм при частоте 1,035 кГц и амплитуде 1 мкм.

Выявленные закономерности открывают возможности практического применения метода акустического разделения ультрадисперсных материалов, имеющего такие важные преимущества, как способность разделения в газовой среде без жидкости, независимость от материала частиц. Возможность разделения при низких резонансных частотах позволяет использовать колебательные поверхности больших размеров и с большим расстоянием между узлами и пучностями, что облегчает сбор фракций порошка. Изменение частоты и амплитуды колебаний способствует регулированию размера частиц в разделенных фракциях. Это открывает новые возможности получения нанопорошков с малым разбросом размеров частиц, что, в свою очередь, увеличит эффективность их применения в материаловедении и микроэлектронике.

Материалы публикации подготовлены с использованием оборудования ЦКП "Научные приборы" ФГБОУ ВО "Бурятский государственный университет имени Доржк Банзарова".

\section{ЛИТЕРATУPA / REFERENCE}

1. Калашников С.В., Раднаев А.Р., Номоев А.В., Дзидзигури Э.Л. Технологические и прочностные свойства силумина, модифицированного наночастицами диоксида кремния // Металлы. 2017. № 1. С. 37-42.

2. Калашников С.В., Лыгденов В.Ц., Номоев А.В., Татарникова Н.Н. Термомеха- нические свойства кремнийорганического компаунда, содержащего микро- и наноразмерные порошки различных веществ // Нанотехнологии: наука и производство. 2015. № 3. С. 25-33.

3. Бардаханов С.П., Ким А.В., Лысенко В.И., Номоев А.В., Труфанов Д.Ю., Лыгденов В.Ц. Влияние нанопорошка Таркосила на свойства эмалей // Лакокрасочные материалы и их применение. 2009. № 7. С. 32.

4. Бубенчиков М.А., Потекаев А.И. Седиментация наночастиц в поле центробежных сил // Известия высших учебных заведений. Физика. 2011. № 2. С. 74-80.

5. Дзидзигури Э.Л., Калашников С.В., Номоев А.В. Дифференциация наночастиц диоксида кремния по размерам в поле центробежных сил // Российские нанотехнологии. 2014. T. 9. № 9-10. C. 52-54.

6. Dorrestijn M., Bietsch A., Açikalin T. [and etc.]. Chladni Figures Revisited Based on Nanomechanics // Phys. Rev. Lett. 2007. № 98. P. 026102(1-4).

7. Калашников С.В., Номоев А.В., Дзидзигури Э.Л. Использование метода инверсных фигур Хладни для разделения частиц по размерам // Физика и химия обработки материалов. 2014. № 4. С. 68-73.

8. Acikalin T., Ramah A., Garimella S.V. Twodimensional streaming flows induced by resonating thin beams // J.Acoust.Soc. Am. 2003. V. 114. № 4. Pt. 1. P. 1785-1795.

9. Latifi K., Wijaya H., Zhou Q. Motion of Heavy Particles on a Submerged Chladni Plate // Phys. Rev. Lett. 2019. № 122. P. 184301.

10. Sclichting H. Boundary layer theory. - New-York: McCraw-Hill, 1955.

11. Калашников С.В., Номоев А.В., Дармаев Т.Г. Численное моделирование потоков газа над поперечно колеблющейся пластиной в рамках модели разделения наночастиц по размерам // Плазменная эмиссионная электроника. Труды VI международного Крейнделевского семинара "Плазменная эмиссионная электроника". - Улан-Удэ: Изд-во БНЦ СО РАН, 2018. C. 204-208.

12. Van Gerner H.J. Newton vs stokes: competing forces in granular matter. - Enschede: University of Twente, 2009. 145 p.

13. Калашников С.В, Лыгденов В.Ц., Номоев А.В. [и др.]. Разделение наночастиц диоксида кремния по размерам методом фигур Хладни // Актуальные проблемы гуманитарных и естественных наук. 2012. № 8(43). С. 21-28. 